\title{
Development and Evaluation of Species-Specific Biomass Models for Most Common Timber and Fuelwood Species of Bangladesh
}

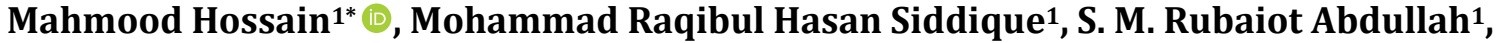 \\ Chameli Saha1, S. M. Zahirul Islam², Md. Zaheer Iqbal'3 , Mariam Akhter ${ }^{4}$ \\ ${ }^{1}$ Forestry and Wood Technology Discipline, Khulna University, Khulna, Bangladesh \\ ${ }^{2}$ Forest Inventory Division, Bangladesh Forest Research Institute, Chittagong, Bangladesh \\ ${ }^{3}$ Deputy Conservator of Forests, Bangladesh Forest Department, Dhaka, Bangladesh \\ ${ }^{4}$ Bangladesh Forest Department, Assistant Conservator of Forest Bangladesh Forest Department, Dhaka, Bangladesh \\ Email: ^mahmoodhossain@hotmail.com,raqibulhasan_fwt@yahoo.com,rubaiot@yahoo.com, chamelifwt@gmail.com, \\ zahir.fid.bfri@yahoo.com,z.iqbal60@gmail.com, mariamakter2002@gmail.com
}

How to cite this paper: Hossain, M., Siddique, M.R.H., Abdullah, S.M.R., Saha, C., Islam, S.M.Z., Iqbal, M.Z., \& Akhter, M. (2020). Development and Evaluation of Species-Specific Biomass Models for Most Common Timber and Fuelwood Species of Bangladesh. Open Journal of Forestry, 10, 172-185.

https://doi.org/10.4236/ojf.2020.101012

Received: December 9, 2019

Accepted: January 17, 2020

Published: January 20, 2020

Copyright (c) 2020 by author(s) and Scientific Research Publishing Inc. This work is licensed under the Creative Commons Attribution International License (CC BY 4.0).

http://creativecommons.org/licenses/by/4.0/

\begin{abstract}
Allometric biomass models are efficient tools to estimate biomass of trees and forest stands in a non-destructive way. Development of species-specific allometric biomass models requires extensive fieldwork and time. Our study aimed to generate species-specific allometric biomass models for the most common fuelwood and timber species of Bangladesh. We also wanted to evaluate the performances of our models relative to the performances of regional and commonly used pan-tropical biomass models. We used semi-destructive method that incorporates tree-level volume, species-specific biomass expansion factor (BEF), and wood density. We considered four base models, 1) Ln (biomass) $=\mathrm{a}+\mathrm{b} \operatorname{Ln}(\mathrm{D}) ; 2) \operatorname{Ln}$ (biomass) $=\mathrm{a}+\mathrm{b} \operatorname{Ln}(\mathrm{H})$; 3) $\operatorname{Ln}$ (Biomass) $=\mathrm{a}$ $+b \operatorname{Ln}\left(\mathrm{D}^{\wedge} 2 \mathrm{H}\right)$; 4) $\operatorname{Ln}$ (Biomass) $=\mathrm{a}+\mathrm{bLn}(\mathrm{D})+\mathrm{cLn}(\mathrm{H})$ to develop species-specific best-fitted models for Total Above-Ground Biomass (TAGB) and stem biomass. The best-fitted model for each species was selected by the lowest value of Akaike Information Criterion (AIC), Residual Standard Error (RSE) and Root Mean Square Error (RMSE). The derived best-fitted models were then evaluated with respect to regional and pan-tropical models using a separate set of observed data. This evaluation was conducted by computing ME (Model Efficiency) and MPE (Model Prediction Error). The best-fitted allometric biomass models have shown higher model efficiency ( 0.85 to 0.99 at scale 1$)$ and the lowest model prediction error $(-8.94 \%$ to $5.27 \%)$ compared to the regional and pan-tropical models. All the examined regional and pan-tropical biomass models showed different magnitude of ME and MPE.
\end{abstract}


Some models showed higher level ( $>0.90$ at scale 1 ) of ME compared to the best-fitted specific species biomass model.

\section{Keywords}

Allometry, Bangladesh, Biomass, Fuelwood, Timber, Pan-Tropical Model, Regional Common Model

\section{Introduction}

Bangladesh has $17.48 \%$ of forestland that ecologically can be classified into three types as tropical evergreen and semi-evergreen forest, tropical moist deciduous forest and mangrove forest (FD, 2017). This country has 163.05 million of population with a density of 1116 person $/ \mathrm{km}^{2}$ that ranks $10^{\text {th }}$ position in the world (World Population Review, 2019). This large population imposes immense pressure on natural resources of this country (Reza \& Sharmin, 2016) that also influences the forestry sector through deforestation and degradation of forestlands (FRA, 2000; World Bank, 2016). Recently, Bangladesh has initiated REDD+ activities to reduce emissions of greenhouse gases from deforestation and degradation, conservation and enhancement of forest-carbon stocks, and sustainable management of forests (FD, 2019). Therefore, success of REDD+ activities depends on authenticated data and information on the existing forest areas and their conditions.

Forest inventory is an integral part of forest management as it provides data and information on trees and forest resources. A total of 19 forest inventories were conducted in Bangladesh by Bangladesh Forest Department since 1960. Overtime, the objectives of forest inventories have been shifted from a focus on volume for timber resources to biomass for carbon-related values to meet the demand of $21^{\text {st }}$ century (FD, 2017). Forest can act as a sink and source of carbon (Canadian Forest Service, 2007). Biomass estimation is an important tool for estimating stock and sequestration of carbon in a forested ecosystem (Golley et al., 1975; Vashum \& Jayakumar, 2012; Mahmood, 2014). Biomass of trees and forest can be estimated by following destructive and non-destructive methods (Somogyi et al., 2007; Picard et al., 2012; Mahmood et al., 2015; Wakawa, 2016; Mahmood et al., 2017). Allometric biomass models are frequently used to estimate tree and forest biomass (Somogyi et al., 2007). Destructive, semi-destructive and non-destructive methods are followed to derive species-specific, regional and pan-tropical allometric biomass models (Ketterings et al., 2001; Chave et al., 2005, 2014; Basuki et al., 2009). Destructive method of biomass model development is more accurate compared to others, but this method is usually discouraged from violating regional and/or national forest management policies (Ketterings et al., 2001).

The multi-species regional and pan-tropical biomass models are commonly used for large-scale biomass estimation (Clark \& Kellner, 2012; Mahmood et al., 
2019a, 2019b, 2019c). The first nationwide forest inventory in Bangladesh was conducted in 2007 where pan-tropical biomass model (Above-ground biomass (tons) $=V^{\star} W^{\star} \mathrm{BEF}$, where $V=$ volume over bark tons ha ${ }^{-1}, W=$ wood density tons $\mathrm{m}^{-3}$ and $\mathrm{BEF}=$ biomass expansion factor) of Brown \& Lugo (1992) was used to estimate the biomass stock in forest areas. This estimation included a common wood density value $\left(0.57 \mathrm{t} \cdot \mathrm{m}^{-3}\right)$ and a fixed biomass expansion factor (6) (FD, 2007) which may result in uncertainty in biomass estimation by considering a lower wood density and a higher fixed value of biomass expansion factor (Penman et al., 2003). During the year 2009, pan-tropical model of Chave et al. (2005) was also used to estimate the biomass and carbon stock of the Sundarbans of Bangladesh (Rahman et al., 2015). The used pan-tropical model is capable to generalize poorly with its polynomial function that results in implausible relationship among biomass and diameter of trees (Sileshi, 2014). In other ways, pan-tropical biomass model of Brown et al. (1989) and Chave et al. (2005) were commonly used to estimate the biomass and carbon stock in plantation and natural forests of Bangladesh like Miah et al. (2009), Ullah \& Al-Amin (2012), Rahman et al. (2015). Numerous studies demonstrated that pan-tropical models generate higher bias in biomass estimation compared to locally developed models (Vieira et al., 2008; Basuki et al., 2009; Kenzo et al., 2009; Ngomanda et al., 2014; Maulana et al., 2016). Therefore, it is recommended to check the bias/ deviation in biomass estimation using the multi-species regional and pan-tropical models for a particular species and forests (Alvarez et al., 2012). Simultaneously, species-specific allometric biomass model may significantly reduce bias in biomass estimation compared to the multi-species regional and commonly used pan-tropical models because they may not able to capture the variability of tree properties (height and diameter at breast height relationship, wood density) caused by ecological and management intervention (Nam et al., 2016; Maulana et al., 2016; Mahmood et al., 2019c). Therefore, this study aimed i) to generate species-specific allometric biomass models for the most common fuelwood and timber species of Bangladesh and ii) to evaluate the performances of the derived best-fitted species-specific models in relation to the performances of multi-species regional and commonly used pan-tropical biomass models.

\section{Materials and Methods}

\subsection{Description of the Study Area}

Sampled trees of this study were collected from the natural patches and plantations of tropical wet evergreen and semi-evergreen forest, tropical moist deciduous forest of Bangladesh during 2018. Bangladesh lies between $20^{\circ} 34^{\prime}$ and $26^{\circ} 38^{\prime}$ north latitude and $88^{\circ} 01^{\prime}$ and $92^{\circ} 41^{\prime}$ east longitude (Figure 1 ). The rainfall ranges from $1500 \mathrm{~mm}$ in the northwest to $5000 \mathrm{~mm}$ in the northeast. Mean monthly maximum temperature is $24^{\circ} \mathrm{C}$ to $37^{\circ} \mathrm{C}$, while mean monthly relative humidity found to vary from $63 \%$ to $83 \%$. Soil texture is silty loam to clay loam and $\mathrm{pH}$ range is 5.5 to 8.3 (Banglapedia, 2014). Albizia procera (Roxb.) Benth., 


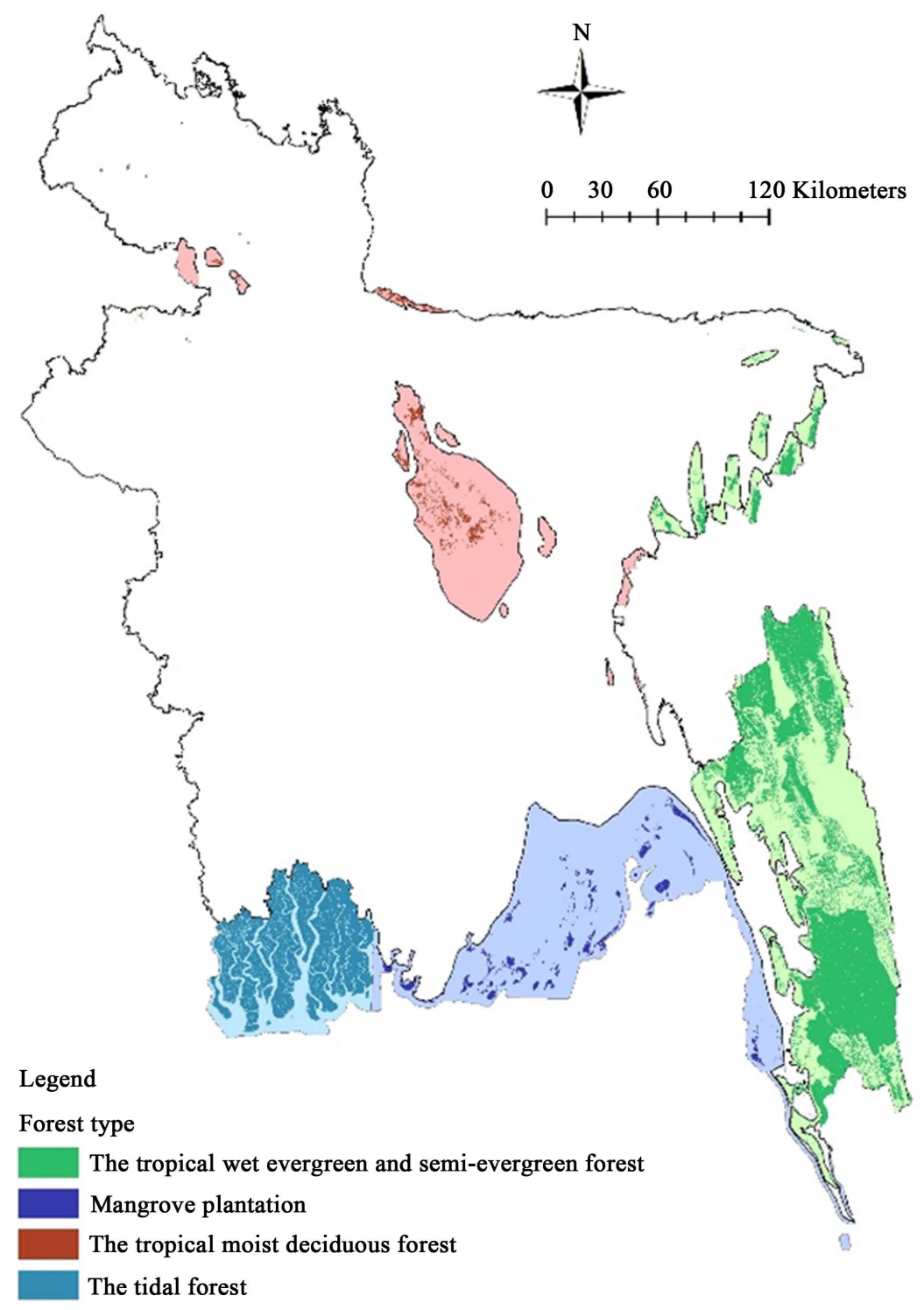

Figure 1. Location of major forest types of Bangladesh.

Albizia richardiana (Voigt) King \& Prain, Dipterocarpus turbinatus C. F. Gaertn., Gmelina arborea Roxb., Lagerstroemia speciosa (L.) Pers., Samanea saman F. Muell., Swietenia macrophylla King, Syzygium grande (Wight) Walp. Tectona grandis $\mathrm{L} . \mathrm{f}$. is the most common timber species found in both natural patches and plantations. While, Acacia auriculiformis A. Cunn. ex Benth., Acacia mangium Willd., Dalbergia sissoo Roxb., Eucalyptus camaldulensis Dehnh., Senna siamea (Lam.) Irwin et Barneby., are the commonest fuelwood species of Bangladesh that mostly restricted in plantation (Das \& Alam, 2001).

\subsection{Biomass Expansion Factor (BEF)}

\subsubsection{Sampling of Trees for Biomass Expansion Factor (BEF)}

Twenty individuals of each studied species, which yielded 280 sample trees, were felled from the natural patches and plantations of the study areas. The species 
were identified using taxonomic key. The sample trees were selected based on subjective judgement to avoid specimens with broken top, hollow trunk, damage caused by natural calamities or animals, and evidence of suppression or disease.

\subsubsection{Field Measurement and Laboratory Analysis}

Total height and DBH of the sampled tree were measured and felled at ground level. The felled trees were separated into leaves, small branches (diameter $<7$ $\mathrm{cm}$ ), bigger branches (diameter $>7 \mathrm{~cm}$ ) and stem. Species wise fresh weight of these components of individual sampled tree were measured and recorded in the field (Picard et al., 2012; Mahmood et al., 2019a, 2019b, 2019c). Ten sub samples $(0.25 \mathrm{~kg})$ of leaf, smaller branch, and ten disk of disk of bigger branches and stem) of individual species were taken randomly from the felled trees. These sub-samples were oven-dried at $105^{\circ} \mathrm{C}$ until a constant weight to estimate the fresh to oven-dry weight conversion factor. The respective conversion factors were used to estimate the oven-dry weight of individual sampled trees (Mahmood et al., 2019a, 2019b, 2019c). Finally, biomass expansion factor (BEF) of individual sampled trees was calculated from the ratio of Total Above-ground Biomass (TAGB) and oven-dry stem biomass, and species-specific average BEF was derived for further use (Taeroe et al., 2015).

$$
\mathrm{BEF}=\frac{\text { Total aboveground biomass }}{\text { Oven-dry stem biomass }} .
$$

\subsection{Allometric Model of Stem and Total Above-Ground (TAGB) Biomass}

\subsubsection{Data Collection and Compilation}

This study used stem volume data of 2490 individuals of 14 most common timber and fuelwood species of Bangladesh that were collected from the natural patches and plantations of tropical wet evergreen and semi-evergreen forest and tropical moist deciduous forest. The mean value with ranges of $\mathrm{DBH}, \mathrm{H}$ and $\mathrm{W}$ of the sampled tree species are presented in Table 1. Bangladesh Forest Research Institute collected the stem volume data from the natural patches and plantations of tropical evergreen and semi-evergreen forest, tropical moist deciduous forest of Bangladesh. Stem biomass ( $\mathrm{kg}$ ) of individual sampled tree was estimated from their stem volume $\left(\mathrm{m}^{3}\right)$ and wood density $(\mathrm{W})\left(\mathrm{kg} \cdot \mathrm{m}^{-3}\right)$ value of the respective tree species as derived by Sattar et al. (1999). TAGB of individual trees was estimated from the stem biomass and species-specific mean BEF.

\subsubsection{Allometric Model Development and Evaluation}

The independent variables ( $\mathrm{D}$ and $\mathrm{H}$ ) and dependent variables (Stem biomass and TAGB) were transformed to Ln (natural logarithm) to improve the linearity and homoscedasticity. Tree volume data was collected in two different occasions. A total of 2490 sample trees (data set A) were selected to derive species-specific allometric biomass model. While, data set B contained 614 individual which was used to validate the derived best-fitted model and comparison with regional and 
Table 1. List of studied species and their wood density, range of DBH and Height, sample number in data set $\mathrm{A}$ and $\mathrm{B}$ and mean biomass expansion factor.

\begin{tabular}{ccccccc}
\hline Species & $\begin{array}{c}\text { Wood } \\
\text { density } \\
\left(\mathrm{kg} \cdot \mathrm{m}^{-3}\right)\end{array}$ & $\begin{array}{c}\text { Range of } \\
\text { DBH }(\mathrm{cm})\end{array}$ & $\begin{array}{c}\text { Range of } \\
\text { Height }(\mathrm{m})\end{array}$ & $\begin{array}{c}\text { Data } \\
\text { set A }\end{array}$ & $\begin{array}{c}\text { Data } \\
\text { set B }\end{array}$ & $\begin{array}{c}\text { Mean } \\
\text { BEF } \pm \text { SE }\end{array}$ \\
\hline Acacia auriculiformis & 700 & $3.9-49.4$ & $5.8-25.0$ & 567 & 61 & $1.39 \pm 0.04$ \\
Acacia mangium & 530 & $5.0-41.8$ & $5.5-28.5$ & 260 & 54 & $1.41 \pm 0.01$ \\
Albizia procera & 730 & $6.9-70.0$ & $4.5-22.0$ & 57 & 28 & $1.51 \pm 0.12$ \\
Albizia richardiana & 580 & $5.1-80.5$ & $4.0-32.5$ & 271 & 81 & $1.50 \pm 0.08$ \\
Dalbergia sissoo & 740 & $6.8-39.3$ & $5.5-19.5$ & 56 & 22 & $1.31 \pm 0.05$ \\
Dipterocarpus turbinatus & 619 & $5.5-51.0$ & $5.0-26.0$ & 170 & 44 & $1.47 \pm 0.11$ \\
Eucalyptus camaldulensis & 721 & $4.5-51.2$ & $8.0-30.5$ & 264 & 60 & $1.35 \pm 0.03$ \\
Gmelina arborea & 540 & $8.5-50.0$ & $6.5-22.0$ & 106 & 31 & $1.28 \pm 0.03$ \\
Lagerstroemia speciosa & 595 & $9.5-56.3$ & $6.5-27.0$ & 257 & 54 & $1.49 \pm 0.07$ \\
Samanea saman & 590 & $5.2-39.1$ & $5.9-19.7$ & 57 & 28 & $1.51 \pm 0.14$ \\
Senna siamea & 660 & $7.8-73.2$ & $6.0-25.0$ & 148 & 51 & $1.63 \pm 0.13$ \\
Swietenia macrophylla & 537 & $9.9-90.5$ & $6.5-32.0$ & 149 & 58 & $1.29 \pm 0.03$ \\
Syzygium grande & 673 & $5.0-38.0$ & $5.0-27.0$ & 84 & 30 & $1.63 \pm 0.14$ \\
Tectona grandis & 720 & $7.0-51.0$ & $9.5-26.0$ & 44 & 12 & $1.41 \pm 0.11$ \\
\hline
\end{tabular}

Note: $\mathrm{BEF}=$ Biomass Expansion Factor, $\mathrm{SE}=$ Standard error of mean.

pan-tropical biomass models. We considered four Ln base models, 1) Ln (biomass) $=a+b \operatorname{Ln}(D) ; 2) \operatorname{Ln}$ (biomass) $=a+b \operatorname{Ln}(H) ; 3) \operatorname{Ln}$ (Biomass) $=a+b \operatorname{Ln}$ $\left(\mathrm{D}^{\wedge} 2 \mathrm{H}\right)$; 4) Ln (Biomass) $=\mathrm{a}+\mathrm{bLn}(\mathrm{D})+\mathrm{cLn}(\mathrm{H})$ to develop species-specific allometric biomass models for TAGB and stem biomass according to (Picard et al., 2012). The best-fitted models were selected based on the lowest Akaike Information Criterion (AIC), Residual Standard Error (RSE) and Root Mean Square Error (RMSE); and highest coefficient of determination (Adjusted $\mathrm{R}^{2}$ ) values (Sileshi, 2014; Mahmood et al., 2019a, 2019b, 2019c). Data were analyzed using R (3.2.3) statistical software. A correction factor (CF) was calculated for each equation to minimize the systematic bias during the back transformation to biomass value (Sprugel, 1983). The derived best-fitted TAGB models were compared and evaluated with the multi-species regional and common pan-tropical models (Table 2) in terms of Model Efficiency (ME) and Model Prediction Error (MEP) (Mayer \& Butler, 1993).

\section{Results}

\subsection{Selection of Allometric Model}

Model 4 (Ln (biomass) $=\mathrm{a}+\mathrm{bLn}(\mathrm{D})+\operatorname{Ln}(\mathrm{H}))$ has appeared as best-fit TAGB and stem biomass allometric model for A. auriculiformis, A. procera, A. richardiana, E. camaldulensis, G. arborea, L. speciosa, S. saman, S. siamea and $S$. grande due to its lowest AIC, RSE and RMSE values. While, Model 3 
Table 2. Multi-species regional and commonly used pan-tropical allometric biomass models.

\begin{tabular}{ccc}
\hline Source & Allometric biomass model & Type \\
\hline $\begin{array}{c}\text { Mahmood et al., 2019a } \\
\text { (Tropical moist deciduous forest) }\end{array}$ & Ln (TAGB) $=-2.460+2.171 \mathrm{Ln}(\mathrm{D})+0.367 \mathrm{Ln}(\mathrm{H})+0.161 \mathrm{Ln}(\mathrm{W})$ & $\begin{array}{c}\text { Regional multi-species, } \\
\text { Bangladesh }\end{array}$ \\
$\begin{array}{c}\text { Mahmood et al., 2019b } \\
\text { (Tropical evergreen and semi-evergreen forest) }\end{array}$ & Ln (TAGB) $=-6.6937+0.809 \mathrm{Ln}\left(\mathrm{D}^{\wedge} 2 \mathrm{HW}\right)$ & $\begin{array}{c}\text { Regional multi-species, } \\
\text { Bangladesh }\end{array}$ \\
Brown et al. (1989) (Moist) & TAGB $=\exp \left(-2.4090+0.9522 \mathrm{Ln}\left(\mathrm{D}^{\wedge} 2 \mathrm{HW}\right)\right)$ & Pan-tropical \\
Chave et al. (2005) & TAGB $=\exp \left(-2.977+\operatorname{Ln}\left(\mathrm{D}^{\wedge} 2 \mathrm{HW}\right)\right)$ & Pan-tropical \\
Chave et al. (2014) & TAGB $=\exp \left(-2.6986+0.976 \mathrm{Ln}\left(\mathrm{D}^{\wedge} 2 \mathrm{HW}\right)\right)$ & Pan-tropical \\
\hline
\end{tabular}

Note: $\mathrm{TAGB}=$ Total aboveground biomass, $\mathrm{D}=$ Diameter at Breast Height, $\mathrm{H}=$ Total Height, $\mathrm{W}=$ Wood density.

(Ln (biomass) $\left.=\mathrm{a}+\mathrm{bLn}\left(\mathrm{D}^{\wedge} 2 \mathrm{H}\right)\right)$ found to be best-fit TAGB and stem allometric biomass model for $A$. mangium, D. sissoo, D. turbinatus, S. macrophylla and T. grandis considering the model selection criteria (Table 3 and Table 4).

\subsection{Model Evaluation and Comparison}

The model efficiency and model prediction error values of the best-fitted TAGB models of the studies species found to vary from 0.85 to 0.99 (at scale 1) and $-8.94 \%$ to $5.27 \%$ respectively. Lower model efficiency and higher prediction error were observed for $S$. saman, $S$. macrophylla and $A$ procera, while the highest model efficiency and lower prediction error were observed for $A$. mangium, $E$. camaldulensis and T. grandis (Table 5).

The best-fitted TAGB model of all the studied species except $A$. procera, $L$. speciosa and $S$. saman showed higher performance in biomass estimation compared to the regional and commonly used pan-tropical allometric models in relation to model efficiency and model prediction error values. TAGB model of Chave et al. (2014) and Chave et al. (2005) have appeared as more efficient in biomass estimation of $A$. procera and $L$. speciosa respectively. While, TAGB models of Mahmood et al. (2019a) and Chave et al. (2014) can efficiently estimate the biomass of $S$. saman compared to the derived model (Table 5).

\section{Discussion}

Alometric biomass models are important tools to estimate biomass of standing trees and stands (Golley et al., 1975; Basuki et al., 2009) and the accuracy in the estimation depends on model efficiency (Sileshi, 2014). Method of model development, involvement of independent variables and model selection criteria influence the efficiency of allometric biomass model (Sileshi, 2014; Picard et al., 2012). Generally, wood density (W), Diameter at Breast Height (DBH) and total height $(\mathrm{H})$ are considered as independent variables of allometric biomass models (Picard et al., 2012). Wood density of a species varies among the ecoregion (Zanne et al., 2009). This study considered DBH and $\mathrm{H}$ as independent variables. But, $\mathrm{W}$ was not included because the sample trees were collected from the same ecoregion that likely to have similar wood density for a particular species 
Table 3. Species-specific best-fitted allometric biomass model for Total Above-Ground Biomass (TAGB).

\begin{tabular}{|c|c|c|c|c|c|c|}
\hline Species name & Best-fitted allometric biomass model & Adjusted $R^{2}$ & AIC & RSE & RMSE & $\mathrm{CF}$ \\
\hline Acacia auriculiformis & $\operatorname{Ln}($ TAGB $)=-2.459+1.869 \operatorname{Ln}(\mathrm{D})+0.800 \operatorname{Ln}(\mathrm{H})$ & 0.986 & -760.515 & 0.123 & 0.123 & 1.007 \\
\hline Acacia mangium & $\operatorname{Ln}(\mathrm{TAGB})=-3.005+0.923 \operatorname{Ln}\left(\mathrm{D}^{\wedge} 2 \mathrm{H}\right)$ & 0.984 & -276.327 & 0.141 & 0.141 & 1.010 \\
\hline Albizia procera & $\operatorname{Ln}(\mathrm{TAGB})=-1.984+1.911 \operatorname{Ln}(\mathrm{D})+0.572 \operatorname{Ln}(\mathrm{H})$ & 0.969 & -13.870 & 0.205 & 0.205 & 1.021 \\
\hline Albizia richardiana & $\operatorname{Ln}(T A G B)=-2.111+1.832 \operatorname{Ln}(D)+0.648 \operatorname{Ln}(H)$ & 0.972 & -62.744 & 0.214 & 0.214 & 1.023 \\
\hline Dalbergia sissoo & $\operatorname{Ln}(T A G B)=-2.608+0.905 \operatorname{Ln}\left(D^{\wedge} 2 \mathrm{H}\right)$ & 0.985 & -53.709 & 0.145 & 0.145 & 1.010 \\
\hline Dipterocarpus turbinatus & $\operatorname{Ln}(T A G B)=-2.525+0.897 \operatorname{Ln}\left(D^{\wedge} 2 H\right)$ & 0.974 & -67.920 & 0.196 & 0.196 & 1.019 \\
\hline Eucalyptus camaldulensis & $\operatorname{Ln}(T A G B)=-2.663+1.915 \operatorname{Ln}(D)+0.832 \operatorname{Ln}(H)$ & 0.989 & -349.155 & 0.124 & 0.124 & 1.008 \\
\hline Gmelina arborea & $\operatorname{Ln}(T A G B)=-2.421+1.585 \operatorname{Ln}(D)+1.011 \operatorname{Ln}(H)$ & 0.976 & -102.663 & 0.146 & 0.146 & 1.011 \\
\hline Lagerstroemia speciosa & $\operatorname{Ln}(T A G B)=-2.909+1.976 \operatorname{Ln}(D)+0.829 \operatorname{Ln}(H)$ & 0.968 & -199.884 & 0.162 & 0.150 & 1.013 \\
\hline Samanea saman & $\operatorname{Ln}(T A G B)=-2.461+1.933 \operatorname{Ln}(D)+0.660 \operatorname{Ln}(H)$ & 0.984 & -24.594 & 0.167 & 0.167 & 1.014 \\
\hline Senna siamea & $\operatorname{Ln}(T A G B)=-2.597+1.835 \operatorname{Ln}(D)+0.951 \operatorname{Ln}(H)$ & 0.992 & -289.357 & 0.090 & 0.090 & 1.004 \\
\hline Swietenia macrophylla & $\operatorname{Ln}(\mathrm{TAGB})=-2.302+0.894 \operatorname{Ln}\left(\mathrm{D}^{\wedge} 2 \mathrm{H}\right)$ & 0.974 & -89.208 & 0.177 & 0.177 & 1.016 \\
\hline Syzygium grande & $\operatorname{Ln}(T A G B)=-2.713+1.529 \operatorname{Ln}(D)+1.324 \operatorname{Ln}(H)$ & 0.968 & -7.211 & 0.225 & 0.225 & 1.026 \\
\hline Tectona grandis & $\operatorname{Ln}(T A G B)=-2.180+0.875 \operatorname{Ln}\left(\mathrm{D}^{\wedge} 2 \mathrm{H}\right)$ & 0.973 & -25.420 & 0.173 & 0.173 & 1.015 \\
\hline
\end{tabular}

Note: AIC $=$ Akaike Information Criterion, RSE $=$ Residual Standard Error, RMSE $=$ Root Mean Square Error, CF $=$ Correction Factor.

Table 4. Species-specific best-fitted allometric biomass model for Stem.

\begin{tabular}{|c|c|c|c|c|c|c|}
\hline Species name & formula & Adjusted $\mathbf{R}^{2}$ & AIC & RSE & RMSE & $\mathrm{CF}$ \\
\hline Acacia auriculiformis & $\operatorname{Ln}($ Stem $)=-2.787+1.869 \operatorname{Ln}(\mathrm{D})+0.800 \operatorname{Ln}(\mathrm{H})$ & 0.985 & -760.631 & 0.123 & 0.123 & 1.007 \\
\hline Acacia mangium & $\operatorname{Ln}($ Stem $)=-3.327+0.923 \operatorname{Ln}\left(\mathrm{D}^{\wedge} 2 \mathrm{H}\right)$ & 0.984 & -276.356 & 0.141 & 0.141 & 1.010 \\
\hline Albizia procera & $\operatorname{Ln}($ Stem $)=-2.396+1.911 \operatorname{Ln}(\mathrm{D})+0.572 \operatorname{Ln}(\mathrm{H})$ & 0.969 & -13.870 & 0.205 & 0.205 & 1.021 \\
\hline Albizia richardiana & $\operatorname{Ln}($ Stem $)=-2.532+1.832 \operatorname{Ln}(\mathrm{D})+0.648 \operatorname{Ln}(\mathrm{H})$ & 0.972 & -62.742 & 0.214 & 0.214 & 1.023 \\
\hline Dalbergia sissoo & $\operatorname{Ln}($ Stem $)=-2.878+0.904 \operatorname{Ln}\left(\mathrm{D}^{\wedge} 2 \mathrm{H}\right)$ & 0.985 & -53.709 & 0.145 & 0.145 & 1.011 \\
\hline Dipterocarpus turbinatus & $\operatorname{Ln}($ Stem $)=-2.911+0.897 \operatorname{Ln}\left(\mathrm{D}^{\wedge} 2 \mathrm{H}\right)$ & 0.974 & -67.922 & 0.196 & 0.196 & 1.019 \\
\hline Eucalyptus camaldulensis & $\operatorname{Ln}($ Stem $)=-2.963+1.915 \operatorname{Ln}(\mathrm{D})+0.832 \operatorname{Ln}(\mathrm{H})$ & 0.989 & -349.194 & 0.124 & 0.124 & 1.008 \\
\hline Gmelina arborea & $\operatorname{Ln}($ Stem $)=-2.668+1.585 \operatorname{Ln}(\mathrm{D})+1.011 \operatorname{Ln}(\mathrm{H})$ & 0.976 & -102.663 & 0.146 & 0.146 & 1.011 \\
\hline Lagerstroemia speciosa & $\operatorname{Ln}($ Stem $)=-3.307+1.976 \operatorname{Ln}(\mathrm{D})+0.829 \operatorname{Ln}(\mathrm{H})$ & 0.968 & -199.882 & 0.162 & 0.162 & 1.013 \\
\hline Samanea saman & $\operatorname{Ln}($ Stem $)=-2.873+1.933 \operatorname{Ln}(\mathrm{D})+0.660 \operatorname{Ln}(\mathrm{H})$ & 0.984 & -24.594 & 0.167 & 0.167 & 1.014 \\
\hline Senna siamea & $\operatorname{Ln}($ Stem $)=-3.057+1.835 \operatorname{Ln}(\mathrm{D})-0.951 \operatorname{Ln}(\mathrm{H})$ & 0.992 & -289.374 & 0.090 & 0.008 & 1.004 \\
\hline Swietenia macrophylla & $\operatorname{Ln}($ Stem $)=-2.556+0.894 \operatorname{Ln}\left(\mathrm{D}^{\wedge} 2 \mathrm{H}\right)$ & 0.974 & -89.208 & 0.177 & 0.177 & 1.016 \\
\hline Syzygium grande & $\operatorname{Ln}($ Stem $)=-3.202+1.529 \operatorname{Ln}(\mathrm{D})+1.324 \operatorname{Ln}(\mathrm{H})$ & 0.968 & -7.211 & 0.225 & 0.225 & 1.026 \\
\hline Tectona grandis & $\operatorname{Ln}($ Stem $)=-2.524+0.875 \operatorname{Ln}\left(\mathrm{D}^{\wedge} 2 \mathrm{H}\right)$ & 0.973 & -25.420 & 0.173 & 0.173 & 1.015 \\
\hline
\end{tabular}

Note: AIC $=$ Akaike Information Criterion, RSE $=$ Residual Standard Error, RMSE $=$ Root Mean Square Error, $\mathrm{CF}=$ Correction Factor. 
Table 5. Comparison of species-specific best-fitted TAGB model with regional and commonly used pan-tropical allometric biomass models.

\begin{tabular}{|c|c|c|c|c|c|c|c|c|c|c|c|c|}
\hline \multirow{2}{*}{ Species } & \multicolumn{2}{|c|}{ Best-fitted } & \multicolumn{2}{|c|}{$\begin{array}{l}\text { Mahmood et al. } \\
\text { (2019a) }\end{array}$} & \multicolumn{2}{|c|}{$\begin{array}{l}\text { Mahmood et al. } \\
\text { (2019b) }\end{array}$} & \multicolumn{2}{|c|}{$\begin{array}{c}\text { Brown et al. } \\
\text { (1989) }\end{array}$} & \multicolumn{2}{|c|}{$\begin{array}{l}\text { Chave et al. } \\
\text { (2005) }\end{array}$} & \multicolumn{2}{|c|}{$\begin{array}{l}\text { Chave et al. } \\
\text { (2014) }\end{array}$} \\
\hline & ME & $\begin{array}{l}\text { MPE } \\
(\%)\end{array}$ & ME & $\begin{array}{l}\text { MPE } \\
(\%)\end{array}$ & ME & $\begin{array}{l}\text { MPE } \\
(\%)\end{array}$ & ME & $\begin{array}{l}\text { MPE } \\
(\%)\end{array}$ & ME & $\begin{array}{l}\text { MPE } \\
(\%)\end{array}$ & ME & $\begin{array}{l}\text { MPE } \\
(\%)\end{array}$ \\
\hline Acacia auriculiformis & 0.95 & 2.62 & 0.89 & -31.15 & 0.83 & 55.66 & 0.61 & 28.01 & 0.75 & 6.21 & 0.70 & 15.78 \\
\hline Acacia mangium & 0.99 & 0.12 & 0.93 & -15.56 & 0.86 & 63.51 & 0.84 & 25.46 & 0.94 & 1.59 & 0.91 & 12.11 \\
\hline Albizia procera & 0.94 & -8.94 & 0.82 & -27.28 & 0.94 & 23.57 & 0.88 & 6.34 & 0.94 & -10.86 & 0.93 & -3.30 \\
\hline Albizia richardiana & 0.94 & 3.18 & 0.91 & -9.83 & 0.74 & 44.05 & 0.51 & 30.97 & 0.71 & 11.83 & 0.64 & 20.20 \\
\hline Dalbergia sissoo & 0.96 & -3.29 & 0.83 & -17.59 & 0.91 & 53.72 & 0.83 & 31.73 & 0.94 & 10.36 & 0.90 & 19.75 \\
\hline Dipterocarpus turbinatus & 0.98 & 1.44 & 0.87 & -23.32 & 0.90 & 39.40 & 0.84 & 13.83 & 0.93 & -5.96 & 0.90 & 2.74 \\
\hline Eucalyptus camaldulensis & 0.99 & -1.79 & 0.72 & -38.68 & 0.93 & 49.10 & 0.84 & 27.21 & 0.90 & 6.60 & 0.89 & 15.64 \\
\hline Gmelina arborea & 0.96 & 2.00 & 0.90 & 0.28 & 0.10 & 52.10 & 0.15 & 30.07 & 0.63 & 8.67 & 0.45 & 18.10 \\
\hline Lagerstroemia speciosa & 0.96 & 5.27 & 0.81 & -15.99 & 0.94 & 28.71 & 0.87 & 16.65 & 0.94 & -0.72 & 0.92 & 6.89 \\
\hline Samanea saman & 0.85 & -6.55 & 0.86 & -4.41 & 0.84 & 18.08 & 0.78 & 8.61 & 0.82 & -7.01 & 0.81 & -0.19 \\
\hline Senna siamea & 0.97 & 0.89 & 0.70 & -41.58 & 0.94 & 28.43 & 0.96 & -1.44 & 0.95 & -20.39 & 0.97 & -12.01 \\
\hline Swietenia macrophylla & 0.93 & 4.66 & 0.84 & -35.00 & 0.90 & -10.02 & 0.91 & -6.43 & 0.89 & -16.22 & 0.91 & -12.09 \\
\hline Syzygium grande & 0.94 & 0.66 & 0.72 & -37.67 & 0.92 & 28.26 & 0.90 & 1.28 & 0.95 & -17.29 & 0.94 & -9.11 \\
\hline Tectona grandis & 0.99 & -1.33 & 0.65 & -36.10 & 0.97 & 7.45 & 0.84 & 7.88 & 0.94 & 2.20 & 0.91 & 4.65 \\
\hline
\end{tabular}

Note: $\mathrm{ME}=$ Model efficiency, $\mathrm{MPE}=$ Model prediction error.

(Kusmana et al., 2018). Moreover, $\mathrm{W}$ is not recommended as independent variable for species-specific allometric models development due to its lower performance and robustness in use (Njana et al., 2016). Inclusion of more than one independent variable likely to increase the efficiency of allometric models to capture more variabialities (Ketterings et al., 2001; Chave et al., 2005). Our study showed that models with $\mathrm{H}$ and $\mathrm{DBH}$ have higher efficiency for all the studied species and similar findings were also reported by Rutishauser et al. (2013); Kusmana et al. (2018) and Khushi et al. (2019). Allometric models with single independent variable (DBH) are robust in the field measurement and biomass estimation (Ketterings et al., 2001; Chave et al., 2014; Istrefi et al., 2019). But, DBH as single independent variable has shown lower efficiency in model selection parameters of this study.

Model validation is an important stage to precribe best-fitted allometric biomass model for a group of species or single species (Sileshi, 2014). Different predictive performance (goodness-of-fit) statistics like ME, MPE, Roor Mean Squared Relative Prediction Error, graphical presentation of 1:1 line etc. are followed to evaluate performance of best-fitted models (Makungwa et al., 2013; Sileshi, 2014; Huy et al., 2016; Mahmood et al., 2019a, 2019b, 2019c). Best-fitted model of the studied species (except A. procera, S. saman and L. speciosa) showed higher predictive performance in biomass estimation compared to the 
regional and pan-tropical models. The variation in estimated biomass may be due to the differences in tree species, climatic conditions, site conditions, forest types with its composition and management practices which ultimately influence the architecture of tree and biomass partitioning (Poorter et al., 2006; Iida et al., 2011; Mugasha et al., 2016; Nam et al., 2016). Development of species-specific allometric models is quite laborious and time-consuming efforts (Picard et al., 2012). Therefore, regional and pan-tropical allometric biomass models are frequently used to estimate biomass of tree species those do not have species-specific model (Chave et al., 2014). Such application of regional or pantropical models may produce higher variation in biomass estimation compared to species specific allometric biomass models (Ketterings et al., 2001; Ngomanda et al., 2014). However, the species-specific best-fitted allometric models of A. procera, L. speciosa and $\mathcal{S}$. saman in this study showed lower efficiency compared to some regional and pan-tropical models. Therefore, the regional and pan-tropical biomass models can be used to estimate the species-specific biomass prior checking the range of variation generated by using those (Alvarez et al., 2012).

\section{Conclusion}

Allometric biomass models with DBH and $\mathrm{H}$ showed higher efficiency in model selection parameters for all the studied species. Most of the best-fitted biomass models showed higher model efficiency and lower model prediction error compared to the regional and pan-tropical models. Our study suggests using species-specific allometric models for biomass estimation for higher accuracy. In absence of species-specific models, ME and MPE need to be checked for the regional- and pan-tropical models to reduce uncertainties in large scale biomass estimation.

\section{Acknowledgements}

We greatly acknowledge the financial support of Food and Agriculture Organization of the United Nations through GCP/BGD/058/USA (LOA Code: FAOBGDLOA 2017-008) to accomplish the field and laboratory work. We also greatly acknowledge the Bangladesh Forest Research Institute for the sharing of volume data of the studied species. Finally, we like to thank Forestry and Wood Technology Discipline, Khulna University for their logistic supports during the laboratory analysis.

\section{Conflicts of Interest}

The authors declare no conflicts of interest regarding the publication of this paper.

\section{References}

Alvarez, E., Rodríguez. L., Duque, A., Saldarriaga, J., Cabrera, K., de las Salas, G., del Valle, I., Lema, A., Moreno, F., \& Orrego, S. (2012). Tree Above-Ground Biomass Al- 
lometries for Carbon Stocks Estimation in the Natural Forests of Colombia. Forest Ecology and Management, 267, 297-308. https://doi.org/10.1016/j.foreco.2011.12.013

Banglapedia (2014). Climate of Bangladesh. http://en.banglapedia.org/index.php?title=Climate

Basuki, T. M., van Laake, P. E., Skidmore, A. K., \& Hussin, Y. A. (2009). Allometric Equations for Estimating the Above-Ground Biomass in Tropical Lowland Dipterocarp Forests. Forest Ecology and Management, 257, 1684-1694. https://doi.org/10.1016/j.foreco.2009.01.027

Brown, S., \& Lugo, A. E. (1992). Above Ground Biomass Estimates for Tropical Moist Forests of the Brazilian Amazon. Interciencia, 17, 8-18.

Brown, S., Gillespie, A. J. R., \& Lugo, A. E. (1989). Biomass Estimation Method for Tropical Forests with Applications to Forest Inventory Data. Forest Science, 35, 881-902.

Canadian Forest Service (2007). Is Canada's Forest a Carbon Sink or Source? Ottawa: Natural Resources Canada, Canadian Forest Service.

Chave, J., Andalo, C., Brown, S., Cairns, M. A., Chambers, J. Q., Eamus, D., Fölster, H., Fromard, F., Higuchi, N., Kira, T., Lescure, J. P., Nelson, B. W., Ogawa, H, Puig H., Riera, B., \& Yamakura, T. (2005). Tree Allometry and Improved Estimation of Carbon Stocks and Balance in Tropical Forests. Oecologia, 145, 87-99. https://doi.org/10.1007/s00442-005-0100-x

Chave, J., Réjou-Méchain, M., Búrquez, A., Chidumayo, E., Colgan, M. S., Delitti, W. B., Duque, A., Eid, T., Fearnside, P. M., Goodman, R. C., Henry, M., Martínez-Yrízar, A., Mugasha, W. A., Muller-Landau, H. C., Mencuccini, M., Nelson, B. W., Ngomanda, A., Nogueira, E. M., Ortiz-Malavassi, E., Pélissier, R., Ploton, P., Ryan, C. M., Saldarriaga J. G., \& Vieilledent, G. (2014). Improved Allometric Models to Estimate the Aboveground Biomass of Tropical Trees. Global Change Biology, 10, 3177-3190. https://doi.org/10.1111/gcb.12629

Clark, D., \& Kellner, J. R. (2012). Tropical Forest Biomass Estimation and the Fallacy of Misplaced Concreteness. Journal of Vegetation Science, 23, 1191-1196. https://doi.org/10.1111/j.1654-1103.2012.01471.x

Das, D. K., \& Alam, M. K. (2001). Trees of Bangladesh. Chittagong, Bangladesh: Bangladesh Forest Research Institute.

FD (2007). National Forest and Tree Resources Assessment. Dhaka, Bangladesh: Bangladesh Forest Department.

FD (2017). Forest Types of Bangladesh. http://www.bforest.gov.bd/site/page/28615def-835e-4b3a-a412-da954f55a943/-

FD (2019). REDD+ Readiness Roadmap. http://www.bforest.gov.bd/site/page/69f77f4a-4a54-4d40-9464-a45a7fa7a13b/-

FRA (2000). Forest Resources of Bangladesh: Country Report. http://www.fao.org/3/ad104e/AD104E00.htm\#TopOfPage

Golley, B. F., Mc Ginnis, T. J., Clements, G. R., Child, I. G., \& Duever, J. M. (1975). Mineral Cycling in a Tropical Moist Forest Ecosystem. Athens: University of Georgia Press.

Huy, B., Kralicek, K., Poudel, K. P., Phìng, V. T., Khoa, P. V., Hung, N. D., \& Temesgen, H. (2016). Allometric Equations for Estimating Tree Aboveground Biomass in Evergreen Broadleaf Forests of Viet Nam. Forest Ecology and Management, 382, 193-205. https://doi.org/10.1016/j.foreco.2016.10.021

Iida, Y., Kohyama, T. S., Kubo, T., Kassim, A. R., Poorter, L., Sterck, F., \& Potts, M. D. (2011). Tree Architecture and Life-History Strategies across 200 Co-Occurring Tropical Tree Species. Functional Ecology, 25, 1260-1268. 
https://doi.org/10.1111/j.1365-2435.2011.01884.x

Istrefi, E., Toromani, E., Çollaku, N., \& Thaçi, B. (2019). Allometric Biomass Equations for Young Trees of Four Broadleaved Species in Albania. New Zealand Journal of Forestry Science, 49, 8. https://doi.org/10.33494/nzjfs492019x51x

Kenzo, T., Furutani, R., Hattori, D., Kendawang, J. J., Tanaka, S., Sakurai, K., \& Ninomiya, I. (2009). Allometric Equations for Accurate Estimation of above-Ground Biomass in Logged-over Tropical Rainforests in Sarawak, Malaysia. Journal of Forest Research, 14, 365-372. https://doi.org/10.1007/s10310-009-0149-1

Ketterings, Q. M., Coe, R., Noordwijk, M. V., Amagau, Y., \& Palm, C. A. (2001). Reducing Uncertainty in the Use of Allometric Biomass Equations for Predicting above-Ground Tree Biomass in Mixed Secondary Forest. Forest Ecology and Management, 146, 199-209. https://doi.org/10.1016/S0378-1127(00)00460-6

Khushi, M. L. R., Mahmood, H., Abdullah, S. M. R., Saha, S., \& Siddique, M. R. H. (2019). Allometric Models for Estimation of above-Ground Biomass of Gmelina arborea Roxb. in Pulpwood Plantations of Bangladesh. Southern Forests, 81, 45-48. https://doi.org/10.2989/20702620.2018.1488209

Kusmana, C., Hidayat, T., Tiryana, T., Rusdiana, O., \& Istomo (2018). Allometric Models for above- and below-Ground Biomass of Sonneratia spp. Global Ecology and Conservation, 15, e00417. https://doi.org/10.1016/j.gecco.2018.e00417

Mahmood, H. (2014). Carbon Pools and Fluxes in Bruguiera parviflora Dominated Naturally Growing Mangrove Forest of Peninsular Malaysia. Wetlands Ecology and Management, 22, 15-23. https://doi.org/10.1007/s11273-013-9318-2

Mahmood, H., Siddique, M. R. H. S., Abdullah, S. M. R., Akhter, M., \& Islam, S. M. Z. (2017). Manual for Building Tree Volume and Biomass Allometric Equation for Bangladesh. Bangladesh Forest Department.

Mahmood, H., Siddique, M. R. H., Abdullah, S. M. R., Costello, L., Henry, M., Iqbal, M. Z., \& Akhter, M. (2019c). Which Option Best Estimates the above-Ground Biomass of Mangroves of Bangladesh: Pantropical or Site- and Species-Specific Models? Wetlands Ecology and Management, 27, 553-569. https://doi.org/10.1007/s11273-019-09677-0

Mahmood, H., Siddique, M. R. H., Costello, L., Birigazzi, L., Abdullah, S. M. R., Henry, M., Siddiqui, M., Aziz, M., Ali, M., Mamun, A., Forhad, M., Akhter, M., Iqbal, M., \& Mondol, F. (2019a). Allometric Models for Estimating Biomass, Carbon and Nutrient Stock in the Sal Zone of Bangladesh. iForest, 12, 69-75.

https://doi.org/10.3832/ifor2758-011

Mahmood, H., Siddique, M. R. H., Islam, S. M. Z., Abdullah, S. M. R., Matieu, H., Iqbal, M. Z., \& Akhter, M. (2019b). Applicability of Semi-Destructive Method to Derive Allometric Model for Estimating Aboveground Biomass and Carbon Stock in the Hill Zone of Bangladesh. Journal of Forestry Research, 1-11.

https://doi.org/10.1007/s11676-019-00881-5

Mahmood, H., Siddique, M. R. H., Saha, S., \& Abdullah, S. M. R. (2015). Allometric Models for Biomass, Nutrients and Carbon Stock in Excoecaria agallocha of the Sundarbans, Bangladesh. Wetlands Ecology and Management, 23, 765-774.

https://doi.org/10.1007/s11273-015-9419-1

Makungwa, S. D., Chittock, A., Skole, D. L., Kanyama-Phiri, G. Y., \& Woodhouse, I. H. (2013). Allometry for Biomass Estimation in Jatropha Trees Planted as Boundary Hedge in Farmers Fields. Forests, 4, 218-233. https://doi.org/10.3390/f4020218

Maulana, S. I., Wibisono, Y., \& Utomo, S. (2016). Development of Local Allometric Equation to Estimate Total Aboveground Biomass in Papua Tropical Forest. Indonesian Journal of Forest Research, 3, 107-118. https://doi.org/10.20886/ijfr.2016.3.2.107-118 
Mayer, D., \& Butler, D. (1993). Statistical Validation. Ecological Modelling, 68, 21-32. https://doi.org/10.1016/0304-3800(93)90105-2

Miah, M. D., Uddin, M. F., Bhuiyan, M. K., Koike, M., \& Shin, M. Y. (2009). Carbon Sequestration by the Indigenous Tree Species in the Reforestation Program in Bangladesh-Aphanamixis polystachya Wall. and Parker. Forest Science and Technology, 5, 62-65. https://doi.org/10.1080/21580103.2009.9656349

Mugasha, W. A., Mwakalukwa, E. E., Luoga, E., Malimbwi, R. E., Zahabu, E., Silayo, D. S., Sola, G., Crete, P., Henry, M., \& Kashindye, A. (2016). Allometric Models for Estimating Tree Volume and Aboveground Biomass in Lowland Forests of Tanzania. International Journal of Forest Research, 2016, Article ID: 8076271. https://doi.org/10.1155/2016/8076271

Nam, V. T., van Kuijk, M., \& Anten, N. P. R. (2016). Allometric Equations for Aboveground and Belowground Biomass Estimations in an Evergreen Forest in Vietnam. PLoS ONE, 11, e0156827. https://doi.org/10.1371/journal.pone.0156827

Ngomanda, A., Engone Obiang, N. L., Lebamba, J., Moundounga Mavouroulou, Q., Gomat, H., Mankou, G. S., Loumeto, J., Midoko Iponga, D., Kossi Ditsouga, F., \& Zinga Koumba, R. (2014). Site-Specific versus Pantropical Allometric Equations: Which Option to Estimate the Biomass of a Moist Central African Forest? Forest Ecology and Management, 312, 1-9. https://doi.org/10.1016/j.foreco.2013.10.029

Njana, M. A., Meilby, H., Eid, T., Zahabu, E., \& Malimbw, R. E. (2016). Importance of Tree Basic Density in Biomass Estimation and Associated Uncertainties: A Case of Three Mangrove Species in Tanzania. Annals of Forest Science, 73, 1073-1087. https://doi.org/10.1007/s13595-016-0583-0

Penman, J., Gytarsky, M., Hiraishi, T., Krug, T., Kruger, D., Pipatti, R., Buendia, L., Miwa, K., Ngara, T., Tanabe, K., \& Wagner, F. (2003). Definitions and Methodological Options to Inventory Emissions from Direct Human-Induced Degradation of Forests and Revegetation of Other Vegetation Types (32 p.). Hayama, Kanagawa: The Institute for Global Environmental Strategies for the IPCC and The Intergovernmental Panel on Climate Change.

Picard, N., Saint-André, L., \& Henry, M. (2012). Manual for Building Tree Volume and Biomass Allometric Equations: From Field Measurement to Prediction. Rome: Food and Agricultural Organization of the United Nations and Montpellier: Centre de Coopération Internationale en Recherche Agronomique pour le Développement.

Poorter, L., Bongers, L., \& Bongers, F. (2006). Architecture of 54 Moist-Forest Tree Species: Traits, Trade-offs, and Functional Groups. Ecology, 87, 1289-1301. https://doi.org/10.1890/0012-9658(2006)87[1289:AOMTST]2.0.CO;2

Rahman, M. M., Kabir, M. E., Akon, A. S. M. J. U., \& Ando, K. (2015). High Carbon Stocks in Roadside Plantations under Participatory Management in Bangladesh. Global Ecology and Conservation, 3, 412-423. https://doi.org/10.1016/j.gecco.2015.01.011

Reza, S., \& Sharmin, D. (2016). A Comparative Study of Environmental Management Strategies in Japan and Bangladesh. Dhaka: University of Dhaka.

Rutishauser, E., Noor'an, F., Laumonier, Y., Halperin, J., Rufi'ie, Hergoualc'h, K., \& Verchota, L. (2013). Generic Allometric Models Including Height Best Estimate Forest Biomass and Carbon Stocks in Indonesia. Forest Ecology and Management, 307, 219-225. ttps://doi.org/10.1016/j.foreco.2013.07.013

Sattar, M. A., Bhattacharjee, D. K., \& Kabir, M. F. (1999). Physical and Mechanical Properties and Uses of Timbers of Bangladesh. Chittagong: Bangladesh Forest Research Institute.

Sileshi, G. W. (2014). A Critical Review of Forest Biomass Estimation Models, Common 
Mistakes and Corrective Measures. Forest Ecology and Management, 329, 237-254. https://doi.org/10.1016/j.foreco.2014.06.026

Somogyi, Z., Cienciala, E., Mäkipää, R., Muukkonen, P., Lehtonen, A., \& Weiss, P. (2007). Indirect Methods of Large Scale Forest Biomass Estimation. European Journal of Forest Research, 126, 197-207. https://doi.org/10.1007/s10342-006-0125-7

Sprugel, D. G. (1983). Correcting for Bias in Log-Transformed Allometric Equations. Ecology, 64, 209-210. https://doi.org/10.2307/1937343

Taeroe, A., Nord-Larsen, T., Stupak, I., \& Raulund-Rasmussen, K. (2015). Allometric Biomass, Biomass Expansion Factor and Wood Density Models for OP42 Hybrid Poplar in Southern Scandinavia. BioEnerg Research, 8, 1332-1343.

https://doi.org/10.1007/s12155-015-9592-3

Ullah, M. R., \& Al-Amin, M. (2012). Above- and below-Ground Carbon Stock Estimation in a Natural Forest of Bangladesh. Journal of Forest Science, 58, 372-379. https://doi.org/10.17221/103/2011-JFS

Vashum, K. T., \& Jayakumar, S. (2012). Methods to Estimate Above-Ground Biomass and Carbon Stock in Natural Forests-A Review. Journal of Ecosystem \& Echography, 2, 116.

Vieira, S. A., Alves, L. F., Aidar, M. P. M., Araújo, L. S., Baker, T., Batista, J. L. F., Campos, M. C. R., Camargo, P. B., Chave, J., Delitti, W. B., Higuchi, N., Honório, E., Joly, C. A., Keller, M., Martinelli, L. A., Mattos, E. A., Metzker, T., Phillips, O. L., Santos, F. A. M., Shimabukuro, M. T., Silveira, M., \& Trumbore, S. E. (2008). Estimation of Biomass and Carbon Stocks: The Case of the Atlantic Forest. Biota Neotropica, 8, 21-29. https://doi.org/10.1590/S1676-06032008000200001

Wakawa, L. D. (2016). Biomass Estimation in Forest Ecosystems-A Review. Journal of Research in Forestry, Wildlife \& Environment, 8, 126-144.

World Bank (2016). World Development Indicators 2016. Washington DC: The World Bank Group.

World Population Review (2019). Bangladesh Population 2019. http://worldpopulationreview.com/countries/bangladesh-population/

Zanne, A. E., Lopez-Gonzalez, G., Coomes, D. A., Ilic, J., Jansen, S., Lewis, S. L., Miller, R. B., Swenson, N. G., Wiemann, M. C., \& Chave, J. (2009). Global Wood Density Database. Dryad. http://hdl.handle.net/10255/dryad.235 